\title{
An Effective Method for Diagnosing Appendicitis in Children: Appendicitis Scoring System
}

\author{
Yusuf Atakan Baltrak, Seniha Esin Söğüt, Onursal Varlıklı \\ Department of Pediatric Surgery, University of Health Sciences Kocaeli Derince Training and \\ Research Hospital, Kocaeli, Turkey
}

\section{ABSTRACT}

Objectives: This study aims to examine the diagnostic values of physical examination, laboratory and imaging methods of pediatric patients, who were hospitalized, followed up and operated in the pediatric surgery clinic of our hospital with the diagnosis of acute appendicitis, to investigate a simple and useful scoring system that can be formed from those with the highest diagnostic value, and to compare scoring systems concerning their effectiveness in diagnosing appendicitis and reducing negative appendectomy rates.

Methods: The study group consisted of patients hospitalized and treated at the pediatric surgery clinic with a preliminary diagnosis of acute appendicitis between January 2016 and January 2019. The cases were divided into two different groups as appendicitis group (AG) and non-appendicitis group (NAG). Alvarado Appendicitis Score (AAS), Pediatric Appendicitis Score (PAS) and Tzanakis Appendicitis Score (TAS) of the patients were calculated retrospectively in both groups.

Please cite this article as: Baltrak YA, Söğüt SE, Varlıklı O. An Effective Method for Diagnosing Appendicitis in Children: Appendicitis Scoring System. Anatol J Family Med 2020;3(2):158-162.

Address for correspondence: Dr. Yusuf Atakan Baltrak. Department of Pediatric Surgery, University of Health Sciences Kocaeli Derince Training and Research Hospital, Kocaeli, Turkey

Phone: +90 5054897503

E-mail:

dratakanbaltrak@gmail.com

Received Date: 10.02 .2020

Accepted Date: 30.04 .2020

Published online: 21.08 .2020

@Copyright 2020 by Anatolian Journal of Family Medicine -

Available online at www.anatoljfm.org

OPEN ACCESS

Results: The difference between the PAS, AAS and TAS of AG and NAG was statistically significant $(p=0.042$, $p=0.021$ and $p=0.021$, respectively). The rate of neutrophil-to-lymphocyte ratio of $>3.5$ in $53(77.9 \%)$ of the AG group and $33(63.4 \%)$ of NAG was higher than 3.5 ( $p=0.030)$.

Conclusion: This study has shown that appendicitis scoring methods are as effective as imaging techniques in the diagnosis of appendicitis, which is the most common cause of surgical abdominal pain in children admitting to emergency care units due to abdominal pain, as well as in reducing the negative appendectomy rates.

Keywords: Appendectomy, appendicitis, methods, scoring methods

\section{INTRODUCTION}

Acute appendicitis is known as one of the most typical reasons for severe abdominal pain, with a $1.1 \%$ chance of occurring and a $7 \%$ total lifetime risk. ${ }^{[1]}$ While acute appendicitis is the most prevalent cause of pediatric abdominal pain, it is difficult to identify in young populations, especially children ${ }^{[2]}$ Although ultrasonography (USG) is the best alternative in the differential acute appendicitis diagnosis, there is still variability in specificity, sensitivity, and accuracy of diagnostic in adult and pediatric age groups. ${ }^{[3]}$ Scoring systems were designed to evaluate appendicitis and to assist in its clinical evaluation. ${ }^{[4]}$ Furthermore, appendicitis scoring systems may prevent acute appendicitis in the pediatric population and reduce negative appendectomy rates. ${ }^{[5]}$

This study aims to examine the diagnostic values of physical examination, laboratory and imaging methods of pediatric patients, who were hospitalized, followed up and operated in our hospital's pediatric surgery clinic with the diagnosis of acute appendicitis, to inves- 
tigate an effortless and useful scoring system, which can be formed from those with the highest diagnostic value, and to compare scoring methods concerning their effectiveness in diagnosing appendicitis and reducing negative appendectomy rates.

\section{METHOD}

In this cross-sectional study, we evaluated the patients, who were hospitalized, followed up and treated in our hospital's pediatric surgery clinic with a preliminary diagnosis of acute appendicitis, with appendicitis scoring method.

The study group consisted of patients hospitalized and treated at the pediatric surgery clinic with a preliminary acute appendicitis diagnosis between January 2016 and January 2019. Cases with abdominal pain due to other causes other than the preliminary diagnosis of appendicitis and patients, whose data required for the appendicitis scoring system could not be obtained in full, were excluded from this study. Finally, we included 120 patients in this study. The medical files of all included patients were examined and scored based on appendicitis scoring systems. The used criteria for the evaluation of scoring systems were as follows: Diagnostic accuracy (DA), negative predictive value (NPV), positive predictive value (PPV), specificity, and sensitivity.

We divided cases into two different groups: the appendicitis group (AG) and the non-appendicitis group (NAG). Patients who went through surgery and whose histopathological diagnosis was reported as appendicitis were included in the AG, whereas other patients who were discharged without undergoing surgery and whose histopathological diagnosis was not appendicitis were included in NAG.
Alvarado Appendicitis Score (AAS), Pediatric Appendicitis Score (PAS) and Tzanakis Appendicitis Score (TAS) of the patients were calculated retrospectively in both groups (Table 1). ${ }^{[2]}$

Leukocyte count (WBC), neutrophil count (NC), neutrophilto-lymphocyte ratio (NLR), and acute phase reactant $C$ Reactive Protein (CRP) were analyzed. Automatic hematology analyzer (Sysmex ${ }^{\circledR}$ pocH-100i Automated Hematology Analyzer, SN: F6797, Sysmex Corporation, Kobe-Japan) was used for hemogram measurement. In the laboratory examinations, the diagnostic value of NLR was also evaluated concerning acute appendicitis.

All the data were collected in a Microsoft Excel worksheet. We used SPSS software version 25 for our statistical analysis (SPSS Inc., Chicago, IL, USA). Descriptive data was presented in frequency, percentage, mean, standard deviation, median, minimum and maximum. Data distribution was analyzed using Kolmogorov-Smirnov. If the distribution was normal, the Student t-test was used, and if not, the Mann-Whitney $U$ test was used. Categorical values were compared using Chi-Square Tests. Sensitivity, specificity, PPV, NPV and DA criteria were used for the evaluation of scoring systems. A p-value of $<0.05$ was considered statistically significant.

\section{RESULTS}

A total of 120 patients were included in this study. The median age of patients was $12.0(5.0-17.0)$ years. Of the patients, 66 (55.0\%) were female and 54 (45.0\%) were male. The number of patients undergoing appendectomy was 77 (64.1\%). The histopathological diagnosis of 68 (88.3\%) out of 77 patients undergoing appendectomy was reported to be compatible with appendicitis. Histopathological diag-

Table 1. Scoring system for evaluation of acute appendicitis in children

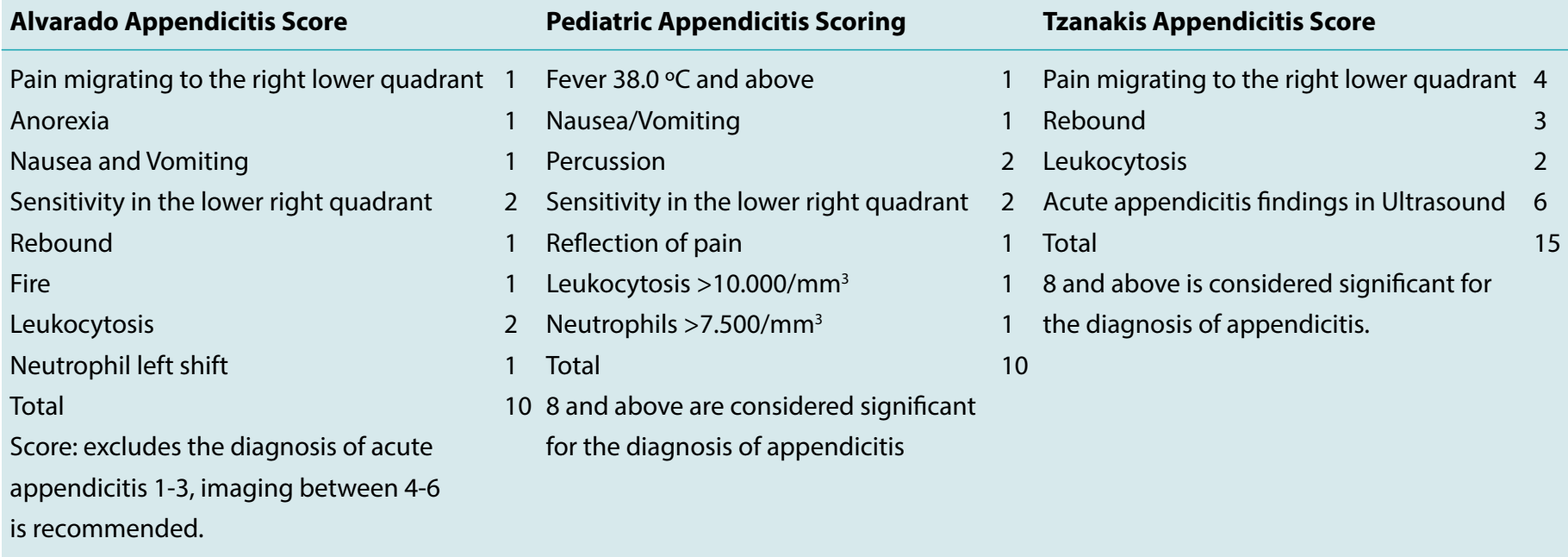


nosis of the $9(11.6 \%)$ patients undergoing appendectomy was not compatible with appendicitis. Of the patients included in this study, 68 (56.6\%) patients whose histopathological diagnosis was compatible with appendicitis were included in the AG, and $52(43.3 \%)$ patients who were discharged without undergoing surgery and whose histopathologic diagnosis was non-appendicitis were included in NAG. Of the 68 patients in the AG, 29 (42.6\%) were male, and 39 (57.3\%) were female (Table 2 ).

Histopathological examination of appendix specimens of the patients in the AG revealed that $26(38.2 \%)$ patients had acute appendicitis accompanied by simple inflammation, 11 (16.1\%) patients had phlegmonous appendicitis, 6 (8.8\%) patients had gangrenous appendicitis, and 5 (7.3\%) patients had perforated appendicitis.

Laboratory results of AG and NAG showed no statistically significant difference between the two groups concerning WBC, NC, and CRP acute phase reactant values that were routinely examined for the appendicitis diagnosis. As the NLR was accepted as higher than 3.5, the difference between AG and NAG was statistically significant $(p=0.030)$ (Table 2 ).

The differences among the PAS, AAS and TAS of AG and NAG groups were statistically significant $(p=0.042, p=0.021$, and $\mathrm{p}=0.021$, respectively) (Table 2 ).

The value of cut-off was accepted as 7 points for the appendicitis diagnosis cases with an AAS of $\geq 7$. Sensitivity, specificity, PPV, NPV, and DA were calculated as $96.0 \%$, $80.1 \%, 88.8 \%, 92.3 \%$, and $90.0 \%$, respectively, for the appendicitis diagnosis cases with a cut-off value of $\geq 7$. The cut-off value for the PAS was accepted as $\geq 8$. Sensitivity, specificity, PPV, NPV, and DA were calculated as $95.0 \%$, $82.1 \%, 89.0 \%, 91.4 \%$, and $89.9 \%$, respectively, for the appendicitis diagnosis cases with a cut-off value of $\geq 8$. The value of cut-off for the TAS was accepted as $\geq 8$. Sensitivity, specificity, PPV, NPV, and DA were calculated as $91.1 \%$, $51.0 \%, 72.9 \%, 80.6 \%$, and $74.3 \%$, respectively, for the appendicitis diagnosis cases with a cut-off value of $\geq 8$. The NLR was accepted as higher than 3.5. Sensitivity, specificity, PPV, NPV, and DA were found to be $73.6 \%, 68.7 \%$, $77.9 \%, 66.4 \%$, and $71.6 \%$, respectively, for the NLR of $>3.5$ (Table 3).

\section{DISCUSSION}

Appendicitis scoring systems reduce the rate of complications due to delay in diagnosis and increase the accuracy of diagnosis. In particular, they further prevent the exposure of pediatric patients to harmful imaging methods. In the previous studies, the AAS specificity has been reported to be low in pediatric patients, whereas PAS has been reported to have a high specificity in pediatric cases in similar studies. New methods that can be used in the diagnosis of appendicitis are being investigated. ${ }^{[2]}$

Negative appendectomy rates have been reported around $30 \%$ in cases where an early operation is preferred. ${ }^{[6]}$ In this study conducted in our clinic, the negative appendectomy rate was $11.6 \%$. A delayed diagnosis may lead to the progression of simple appendicitis to complicated appendicitis and even life-threatening peritonitis. ${ }^{[7,8]}$

As a result, scoring systems created by using the existing diagnostic methods together have begun to be used. Physical examination, history, and the results of imaging Table 2. Appendicitis scores and laboratory measurements between AG and NAG groups

\begin{tabular}{|c|c|c|c|}
\hline & AG $(n=68)$ & NAG $(n=52)$ & $\mathbf{p}$ \\
\hline \multicolumn{4}{|l|}{ Gender } \\
\hline Female & $39(57.3)$ & $27(51.9)$ & \multirow{2}{*}{$0.371^{*}$} \\
\hline Male & $29(42.7)$ & $25(48.1)$ & \\
\hline Number of Leukocytes (×103/uL) & $11.9(4.5-30.6)$ & $9.0(4.5-25.5)$ & $0.343^{+}$ \\
\hline Number of neutrophils ( $\left.\times 10^{3} / \mathrm{uL}\right)$ & $10.5(2.1-2.7)$ & $7.0(1.8-20.8)$ & $0.290^{\dagger}$ \\
\hline Neutrophil/Lymphocyte ratio $\geq 3.5$ & $53(77.9)$ & $33(63.4)$ & $0.030^{*}$ \\
\hline AAS Score & $8.4 \pm 1.7$ & $5.0 \pm 1.9$ & $0.021^{\ddagger}$ \\
\hline PAS Score & $8.4 \pm 1.9$ & $4.8 \pm 1.9$ & $0.042^{\ddagger}$ \\
\hline TAS Score & $11.6 \pm 3.4$ & $7.7 \pm 4.3$ & $0.021^{\ddagger}$ \\
\hline
\end{tabular}

AG: Appendicitis Group; AAS: Alvarado Appendicitis Score; NAG: Non Appendicitis Group; PAS: Pediatric Appendicitis Scoring; TAS: Tzanakis Appendicitis Score. The data were presented as $n(\%)$, median (min-max) and mean \pm standard deviation.

${ }^{*}$ Chi-Square Tests, ${ }^{\dagger}$ Mann-Whitney U test, ${ }^{~}$ Student test-test. 
Table 3. Evaluation of the effectiveness of numerical data of appendicitis scoring studies, ultrasound and neutrophil/ lymphocyte percentage ratios

\begin{tabular}{|c|c|c|c|c|c|}
\hline & Ultrasound (\%) & NLR $\geq 3.5(\%)$ & ALVARADO (\%) & PAS (\%) & TZANAKIS (\%) \\
\hline Specificity & 68.4 & 68.7 & 80.1 & 82.1 & 51.0 \\
\hline NPV & 96.1 & 63.4 & 92.3 & 91.4 & 80.6 \\
\hline Diagnostic Accuracy & 79.1 & 71.6 & 90.0 & 89.9 & 74.3 \\
\hline
\end{tabular}

and laboratory tests are evaluated together in the acute appendicitis diagnosis. It is not possible to diagnose acute appendicitis using only one method. ${ }^{[8,9]}$

PAS is developed to facilitate the diagnosis of acute appendicitis in children. In two studies, in which PAS values of $\geq 7$ were considered significant for surgery, the sensitivity and selectivity of PAS were found to be $97.6-100 \%$ and $96-92 \%$, respectively. ${ }^{[8]}$ PAS and AAS, which is a practical and applicable score, has been used in many studies by modifying its parameters. In this study, we found that AAS and PAS scores are similar in the same patient groups.

TAS consisted of only four parameters with the inclusion of imaging methods in 2005. In the scoring system, which was evaluated over 1 point in total; the risk of appendicitis was found to be above $86 \%$ if the score obtained was $\geq 8$.

Although the use of USG, computed tomography and laparoscopy has become more common, the expected accuracy rates in diagnosis have not been achieved yet. ${ }^{[10]}$ The appendiceal wall thickness of $6 \mathrm{~mm}$ and above is considered the most valuable USG finding in acute appendicitis. In studies evaluating the USG employment in the acute appendicitis diagnosis in children in Turkey, sensitivity and selectivity were reported in the range of $76.4-93.1 \%$ and $80.0-92.2 \%$, respectively. ${ }^{[11,12]}$

In our study, the sensitivity and selectivity of USG were calculated to be $68.4 \%$ and $95.7 \%$, respectively, when the cut-off value was accepted as $6 \mathrm{~mm}$, whereas PPV and NPV were found to be $66.1 \%$ and $96.1 \%$, respectively. We believe that the reason for this low USG sensitivity was that the examination was performed in emergency conditions.

We also found no statistically significant difference between AAS, TAS, and PAS scores in the AG, whereas we found a statistically significant difference between $A G$ and NAG in terms of the appendicitis scores. Appendicitis scor- ing systems were found to be as effective as conventional laboratory tests and imaging methods for the appendicitis diagnosis in pediatric cases in terms of sensitivity, specificity, PPV, NPV, and DA.

In the diagnosis, CRP, WBC, and NLR are particularly valuable and are used as a parameter in scoring systems. These tests can be used in acute appendicitis diagnosis, as well as can provide information about complication development. ${ }^{[13,14]}$ Although imaging methods have been reported to be not effective in reducing negative appendectomy and perforation rates in large population studies, these methods are recommended to avoid delay in diagnosis. Computed tomography is one of the imaging methods used in the differential appendicitis diagnosis in young women and children. However, radiation-related risks in these age groups should not be forgotten. Variables that may occur in the diagnosis while evaluating the computed tomography images by teleradiology in the emergency care unit should be evaluated together with the radiation exposure and high costs due to repeated imaging because of contrast or noncontrast techniques. ${ }^{[15]}$

The limitations of our study are as follows: the relatively small sample size despite the prospective nature of the study, and different physicians deciding for appendectomy for different cases.

\section{CONCLUSION}

In conclusion, this study has shown that appendicitis scoring methods are as effective as imaging techniques in the diagnosis of appendicitis, which is the most prevalent cause of pediatric abdominal pain in the emergency care unit due to abdominal pain, as well as in reducing the negative appendectomy rates.

\section{Disclosures}

Peer-review: Externally peer-reviewed.

Conflict of Interest: None declared. 
Ethics Committee Approval: This study was approved by the research ethics committee of Health Sciences University Kocaeli Derince Training and Research Hospital on 11.04.2019 with approval no. 2019/5.

Authorship Contributions: Concept - Y.A.B., O.V.; Design - S.E.S.; Supervision - Y.A.B., S.E.S.; Materials - O.V., S.E.S.; Data collection \&/or processing - Y.A.B., S.E.S.; Analysis and/or interpretation O.V., Y.A.B.; Literature search - Y.A.B., S.E.S.; Writing - Y.A.B., S.E.S.; Critical review - O.V.

\section{REFERENCES}

1. Ergul E. Heredity and familial tendency of acute appendicitis. Scand J Surg 2007;96(4):290-2.

2. Doria AS, Moineddin R, Kellenberger CJ, Epelman M, Beyene J, Schuh S, Babyn PS, Dick PT. US or CT for Diagnosis of Appendicitis in Children and Adults? A Meta-Analysis. Radiology 2006;241(1):83-94.

3. Reddan T, Corness J, Mengersen K, Harden F. Ultrasound of paediatric appendicitis and its secondary sonographic signs: providing a more meaningful finding. J Med Radiat Sci 2016;63(1):59-66.

4. Schneider C, Kharbanda A, Bachur R. Evaluating appendicitis scoring systems using a prospective pediatric cohort. Ann Emerg Med 2007;49(6):778-84.

5. Arif AS, Amin S, Quamruzzaman SM, Anisur Rahma M. Diagnosing acute appendicitis in children using Alvorado score. Anwer Khan Modern Medical College Journal 2011:2: 11-3.
6. de Castro SM, Ünlü C, Steller EP, van Wagensveld BA, Vrouenraets $B C$. Evaluation of the appendicitis inflammatory response score for patients with acute appendicitis. World J Surg 2012;36(7):1540-5.

7. Coyne JD. Lengthy appendices. J Clin Pathol 2007;60:584.

8. Sarkar A. Congenital absence of the vermiform appendix. Singapore Med J 2012;53:e189.

9. Yaylak F, Algin MC, Gungor Y, Korkmaz M. Atresia of the appendix vermiformis: a rare case of developmental abnormality. World J Gastroenterol 2013;19:122-4.

10. Gray H. Gray's Anatomy: Classic Illustrated Edition. In: Lewis WH, editor. 20 ed. Philadelphia, New York: 2013. p. 693.

11. Marudanayagam R, Williams GT, Rees BI. Review of the pathological results of 2660 appendicectomy specimens. J Gastroenterol 2006:41:745-9.

12. Dingemann J, Ure B. Imaging and the use of scores for the diagnosis of appendicitis in children. Eur J Pediatr Surg 2012:22:195-200.

13. Yang HR, Wang YC, Chung PK, Chen WK, Jeng LB, Chen RJ. Laboratory tests in patients with acute appendicitis. ANZ J Surg. 2006:76:71-4.

14. Goldman RD, Carter S, Stephens D, Antoon R, Mounstephen W, Langer JC. Prospective validation of the pediatric appendicitis score. J Pediatr 2008;153:278-82.

15. Mittal MK, Dayan PS, Macias CG, Bachur RG, Bennett J, Dudley NC, et al. Performance of ultrasound in the diagnosis of appendicitis in children in a multicenter cohort. Acad Emerg Med 2013:20:697-702. 